\title{
Shock-excited radio burst from reconnection outflow jet?
}

\author{
H. Aurass ${ }^{1}$, B. Vršnak ${ }^{2}$, and G. Mann ${ }^{1}$ \\ 1 Astrophysikalisches Institut Potsdam, An der Sternwarte 16, 14482 Potsdam, Germany \\ 2 Hvar Observatory, University of Zagreb, Kačicéva 26, 10000 Zagreb, Croatia
}

Received 9 May 2001 / Accepted 9 November 2001

\begin{abstract}
Models of dynamic (two-ribbon-, arcade) flares involve the formation of a system of standing slow and possibly also fast mode shock waves associated with the fast reconnection process below the erupting filament. These shocks are anticipated theoretically, but are not unambiguously confirmed by observations. In this paper we identify for the first time the radio signature of a fast mode outflow termination shock in a dynamic radio burst spectrogram. The standing fast mode shock is revealed by a zero-drift type II burst recorded between 300 and $400 \mathrm{MHz}$. It started almost 1 hour after the impulsive phase of the 7 April 1997 flare and lasted for more than $30 \mathrm{~min}$. The burst shows a characteristic herringbone fine structure and a band split of $(10 \pm 6) \%$ of the emission frequency. No fundamental-harmonic pattern was observed, and we argue that the feature is fundamental mode emission. Simultaneous imaging observations (H $\alpha$, Yohkoh SXT, SOHO EIT) show a relaxed postflare loop arcade with a bright soft X-ray cusp commonly interpreted as a typical reconnection pattern. Conditions for termination shock formation and excitation of radio emision are investigated. Favourable circumstances for the radio detection of a termination shock in the reconnection outflow are a comparatively large height of the diffusion region, a low plasma to magnetic pressure ratio $\beta$ upstream of the slow shocks, and a small angle between the reconnecting field lines. Finally, we discuss why similar radio signatures are not observed more frequently, and why it appeared so late in the event. We stress the implications and point to some inconsistencies which might be a consequence of commonly practiced ad hoc application of idealized model results to realistic conditions.
\end{abstract}

Key words. Sun: radio radiation; flares - MHD - plasmas - shock waves

\section{Introduction}

The powerful energy release in flares can be attributed to the process of fast reconnection of magnetic fields (e.g. Priest 1982, 1985). This is a complex phenomenon involving plasma kinetic and MHD length and time scales. It is challenging to find a convincing observational evidence, and to give a satisfying theoretical treatment. In the present paper we demonstrate radio evidence of one of the essential features of this process, the fast magnetosonic termination shock in the reconnection outflow jet.

The topological change of the coronal magnetic field provided by reconnection enables a release of the energy stored in the preflare field configuration. In the regime of fast reconnection fields merge typically at a velocity in the order of $10 \%$ of the ambient Alfvén velocity (Priest 1985 and references therein; see also Ugai 1992, 1999; Nitta et al. 2001; Yokoyama \& Shibata 2001). An X-type neutral point is formed where the topology of field lines changes within the so-called diffusion region (DR). The DR is extremely small due to an exceptionally low coronal magnetic diffusivity (high electric conductivity).

Send offprint requests to: H. Aurass, e-mail: haurass@aip.de
We have sketched the plasma-magnetic field configuration of an arcade (dynamic, two-ribbon) flare in Fig. 1. The figure shows an orthogonal cut through the arcade's length axis. The inflowing plasma (arrows pointing toward DR) is squeezed between the merging field line systems and is ejected at high velocity along the thin electric current sheet. Observations are referred to in detail e.g. by Tsuneta (1996, and references therein), by McKenzie \& Hudson (1999), McKenzie (2000) and Yokoyama et al. (2001). Two pairs of slow mode standing MHD shocks (SMSS henceforth) are formed extending from DR and separating the inflow and outflow plasma regions ${ }^{1}$. In the slow mode shocks the inflowing plasma is heated to a high temperature and accelerated to form the outflowing fast jets. It is important to realize that the majority of energy is released at the SMSSs which extend all over the merging magnetic system. The small DR itself serves only to switch-on the fast reconnection process.

1 For the development of numerical simulations, see e.g., Sato \& Hayashi (1979), Forbes \& Priest $(1982,1983)$, Forbes (1988), Ugai (1987a, 1987b, 1992, 1999), Forbes et al. (1989), Forbes \& Malherbe (1991), Magara et al. (1996), Yokoyama \& Shibata (1998, 2001), Nitta et al. (2001). 


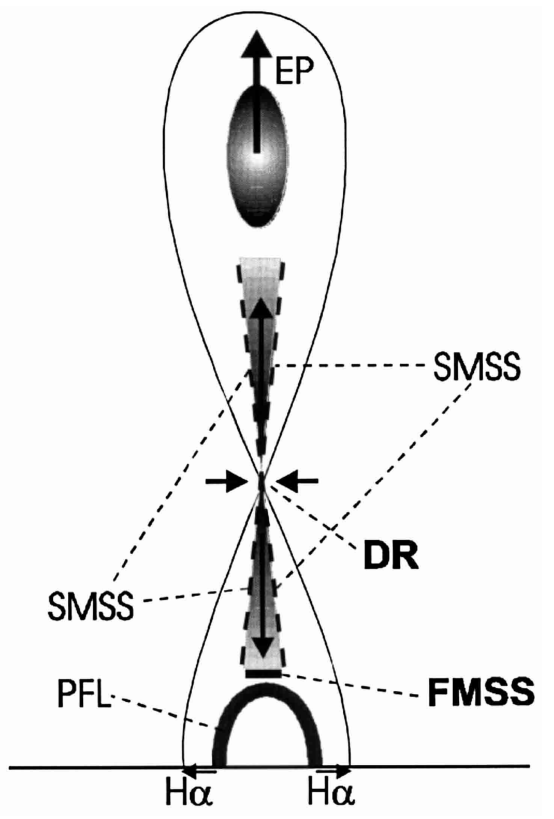

Fig. 1. Simplified dynamic field configuration during a flare. The current sheet is formed under the erupting flux tube (an erupting prominence, EP). Reconnection starts in the diffusion region (DR). Two pairs of slow mode standing shocks (SMSS) expand outward from DR, bounding the hot outflowing jets (thick arrows). If the downflow jet is supermagnetosonic the (perpendicular) fast mode standing shock (FMSS) is formed above the postflare loops (PFL). With ongoing reconnection the $\mathrm{DR}$ rises, the PFL grow, and the $\mathrm{H} \alpha$ ribbons expand laterally.

The SMSSs are sites of strong electric currents (e.g. Sato \& Hayashi 1979) with a current density comparable to DR conditions. They are a potential source of nonthermal particles. Furthermore, the plasma is trapped between the SMSSs. Thus, various kinetic plasma instabilities can be driven (e.g. Somov 1986; Vršnak 1989, and references therein). A wide palette of physical processes becomes feasible quite naturally providing a broad variety of flare appearances.

As sketched in Fig. 1 in dynamical flares both reconnection jets are directed toward an obstacle. For the jet above the DR this is the flux rope surrounding an erupting filament. For the jet below the DR this is the system of dense and inert postflare loops. In this paper we focus on the lower jet which interacts with the postflare loops. The significance of postflare loops as an obstacle is underlined by the sometimes observed loop-top hard X-ray source (Masuda et al. 1994; Shibata et al. 1995). If the outflow jet is supermagnetosonic then in front of the postflare loops a standing fast mode MHD shock will be formed. With respect to this "reconnection outflow termination shock" the DR and the jet are on the upstream side, whereas the postflare loops are on the downstream side. In Fig. 1 we marked the termination shock as FMSS (fast mode standing shock) well below the DR.

At decimeter-meter wavelengths the radio flare emission is excited dominantly by the plasma radiation mechanism (Melrose 1985). It is caused by nonthermal electrons and the interaction of high frequency plasma waves with low frequency wave modes (e.g. ion-acoustic waves, whistlers). Plasma emission can occur at the fundamental and at higher harmonics of the electron plasma frequency thus yielding an information about the density in the source volume.

Broad band radio observations are usually presented as dynamic radio spectrograms (Fig. 2). Due to the properties of plasma emission and the inhomogenity of the solar corona a moving exciter causes a drifting intensity enhancement in the radio spectrogram. Assuming a coronal density model the drift rate can be (under simplified assumptions) transformed into an exciter speed. This is commonly performed for metric type II bursts (Nelson \& Melrose 1985) which are the radio signature of traveling coronal fast mode shock waves.

In analogy with traveling shocks, it can be supposed that the FMSS can appear in the radio spectrogram as a nondrifting type II-like burst. Since the FMSS is located below the primary flare energy release site (i.e. at higher densities), one would expect such a signature occurring near to the frequency range of the very first radio flare signatures. Compared with ordinary type II bursts these are comparatively high frequencies, say about 250. . .300 MHz. Roughly (but not yet systematically, and with a data gap in summer 2000) cross-inspecting the digitally recorded radio spectra of those events which are accompanied by a conventional metric type II burst in the time interval 1996-April 2001 we found one case out of 159 events fitting with these expectations. It is an isolated in time and frequency radio signature in the postflare phase of an event that occurred in AR 8027 on April 7, 1997.

We analyse the observational evidence in Sect. 2. In Sect. 3 we consider the relationship between the plasma inflow parameters and the magnetosonic Mach number of the reconnection outflow jet. The results are discussed in Sect. 4. To ease reading for nonspecialists in reconnection some theoretical background related to Sects. 3 and 4 is briefly surveyed in the Appendix.

\section{The 7 April 1997 flare event}

The event of interest is a $\mathrm{C} 6.9,2 \mathrm{~N}$ flare that took place at about S30E20 on 7 April 1997 between 13:55 and 15:23 UT (NOAA Solar Geophysical Data 638, II). The active region NOAA 8027 consisted of only a few small spots forming a comparatively simple bipolar magnetic configuration.

The event was already treated by several authors. Sterling et al. (2000) discussed the flare as an example of a sigmoid loop pattern seen with the Yohkoh soft X-ray telescope (SXT) and the extreme ultraviolet telescope of the Solar and Heliospheric Observatory (SOHO EIT). Thompson et al. (1998) and Klassen et al. (2000) analyzed the coronal transient wave detected by SOHO EIT. Kaiser et al. (1998) have observed two different type II signatures with the WIND spectrometer (1-13 MHz). The coronograph onboard $\mathrm{SOHO}$ revealed a partial halo 


\section{April 1997 AIP - OSRA}

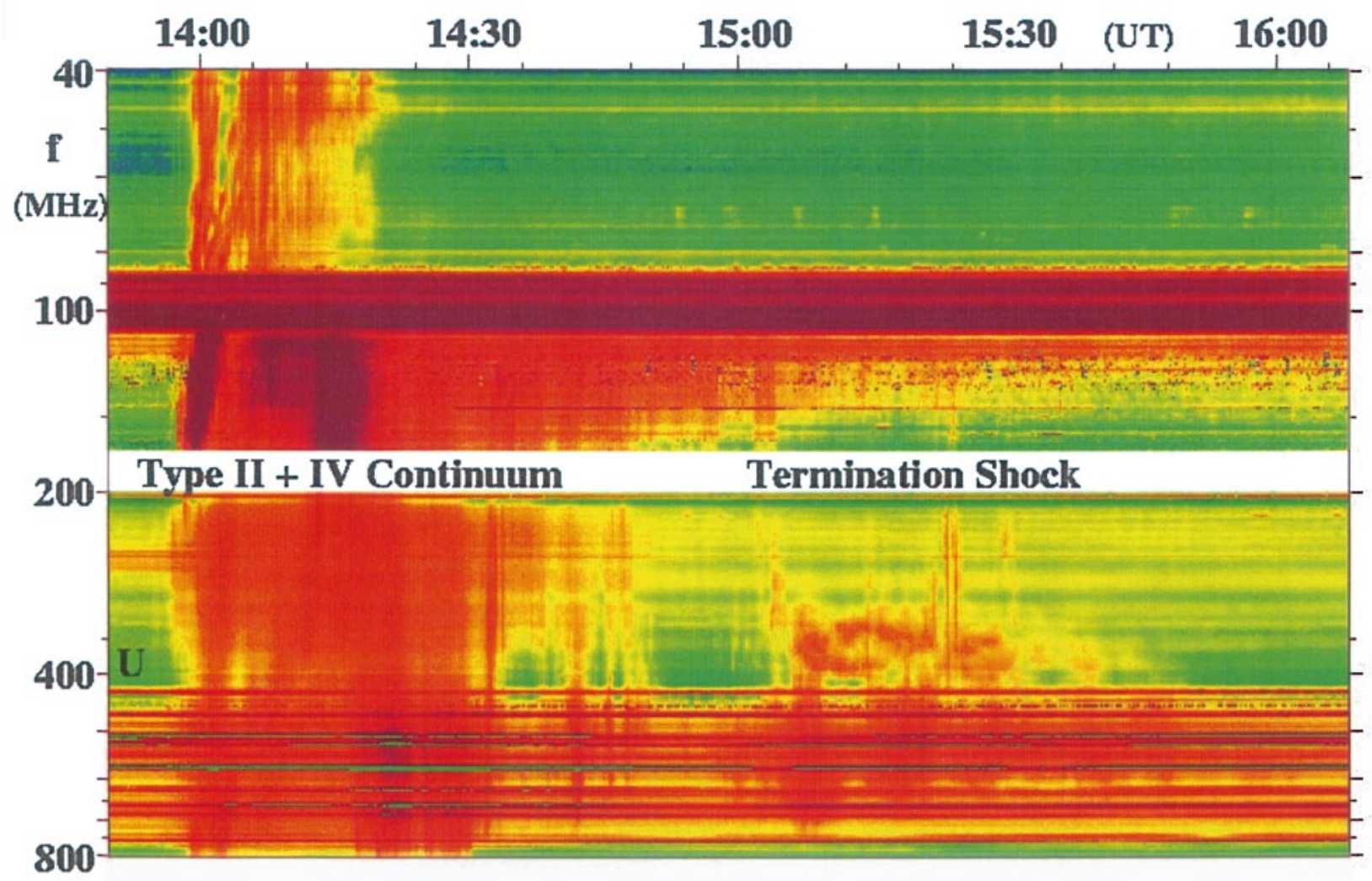

Fig. 2. The 40-800 MHz radio spectrum of the 7 April 1997 flare recorded by the Astrophysical Institute Potsdam, Tremsdorf Solar Radio Observatory. The logarithmic flux density color coded. Time parallel lines are due to terrestrial transmitters. At 88-110 MHz and 170-200 MHz, there are strong local interferences. The effect of interest (enlarged in Fig. 3) starts about 1 hour past the impulsive flare phase. U denotes the rising chain of U-bursts (enlarged in Fig. 4, bottom), type II is the "common" meter wave radio signature of a coronal shock wave.

coronal mass ejection (CME) for which three distinct constant velocity features could be traced (courtesy: the 1997 CME listing of C. St. Cyr).

The $40-800 \mathrm{MHz}$ radio spectrum of the flare burst was observed at Astrophysical Institute Potsdam (Fig. 2) with a time resolution of $0.1 \mathrm{~s}$. The spectrum is presented in logarithmic color-coded arbitrary flux density units. At first glance it shows a sequence of two events which could well be independent ones: a complex $800-40 \mathrm{MHz}$ type II/IV event (13:55-14:50 UT) is followed by a weaker event between 700 and $150 \mathrm{MHz}$ (14:57-16:00 UT). Meter wave imaging observations with the Nançay Radio Heliograph ${ }^{2}$ confirm that the sources of both bursts are located above and to the southeast of AR 8027 (courtesy: French BASS2000 data base in the web).

The impulsive phase radio event starts with a sequence of type $\mathrm{U}$ bursts with a rising turning frequency between 450 and $150 \mathrm{MHz}$ on 13:54 UT. Between 13:58 and 14:20 UT a complex type II/IV burst follows. Until 14:50 UT some brief reappearances of the continuum

\footnotetext{
${ }^{2}$ Available until 15:13 UT.
}

emission are recorded, being strongest from 14:34 to 14:36 UT between 400 and $250 \mathrm{MHz}$.

The second stage of the radio event is shown enlarged in Fig. 3 (the top panel). The dominant feature extending from 14:57 until about 16:00 UT is what we claim to be the radio signature of the outflow termination shock. Yohkoh SXT and SOHO EIT images (Sterling et al. 2000) reveal in the same time an arcade of postflare loops together with a typical reconnection cusp structure above. Unfortunately, no hard X-ray observations are available. The $\mathrm{H} \alpha$ observations (Kanzelhöhe Observatory, courtesy W. Otruba) show the development of some additional substructure in the $\mathrm{H} \alpha$ flare ribbons in the time of termination shock formation. Contemporaneously, the active region filament which disappeared in the impulsive phase showed up again. From the radio spectrum we note in detail:

1. A pair of emission lanes occurs between about 320 and $420 \mathrm{MHz}$. According to our experience, these are comparatively high frequencies for type II burst emission;

2. The emission lanes show almost no frequency drift except a weak positive drift in the final stage; 

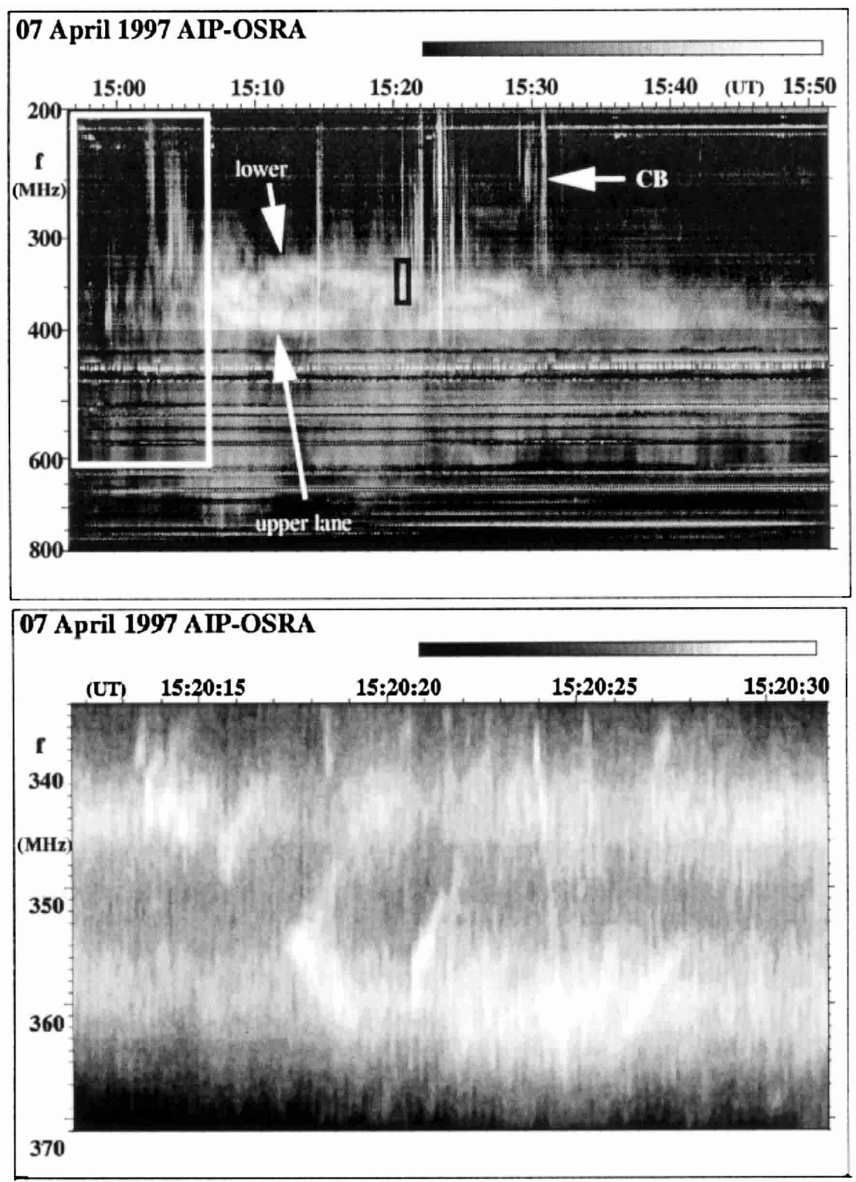

Fig. 3. The termination shock radio signature is shown in the top panel. The bottom panel enlarges the interval defined by the small black box in the top panel. The drifting features are well defined herringbones. The white box encloses the arcpattern and the band split formation (see also Fig. 4 top).

3. Just before the formation of the pair of emission lanes an arc feature (enlargement in Fig. 4 top) appears at about $380 \mathrm{MHz}$. The arc feature can be attributed to the transformation of a large-amplitude wave into a shock. This pecularity of high frequency type II bursts was discovered by Klassen et al. (1999) and theoretically explained by Vršnak \& Lulić (2000);

4. The identification of herringbones (enlargement in Fig. 3 bottom) provides a strong argument for a classification as type II burst;

5. The pair of emission lanes with average maximum frequencies at 380 and $340 \mathrm{MHz}$ can represent the well known split band substructure of type II burst lanes. We obtain a normalized width of the band splitting of $(10 \pm 6) \%$ which is in agreement with reported type II burst split widths (Nelson \& Melrose 1985);

6. The frequency $340 \mathrm{MHz}$ is a level which plays a part already at the very onset of the impulsive phase. In Fig. 4 bottom - enlarged from Fig. 2 - we have encircled a narrowband feature and a diffuse reverse drift burst which both start at $340 \mathrm{MHz}$. We argue that
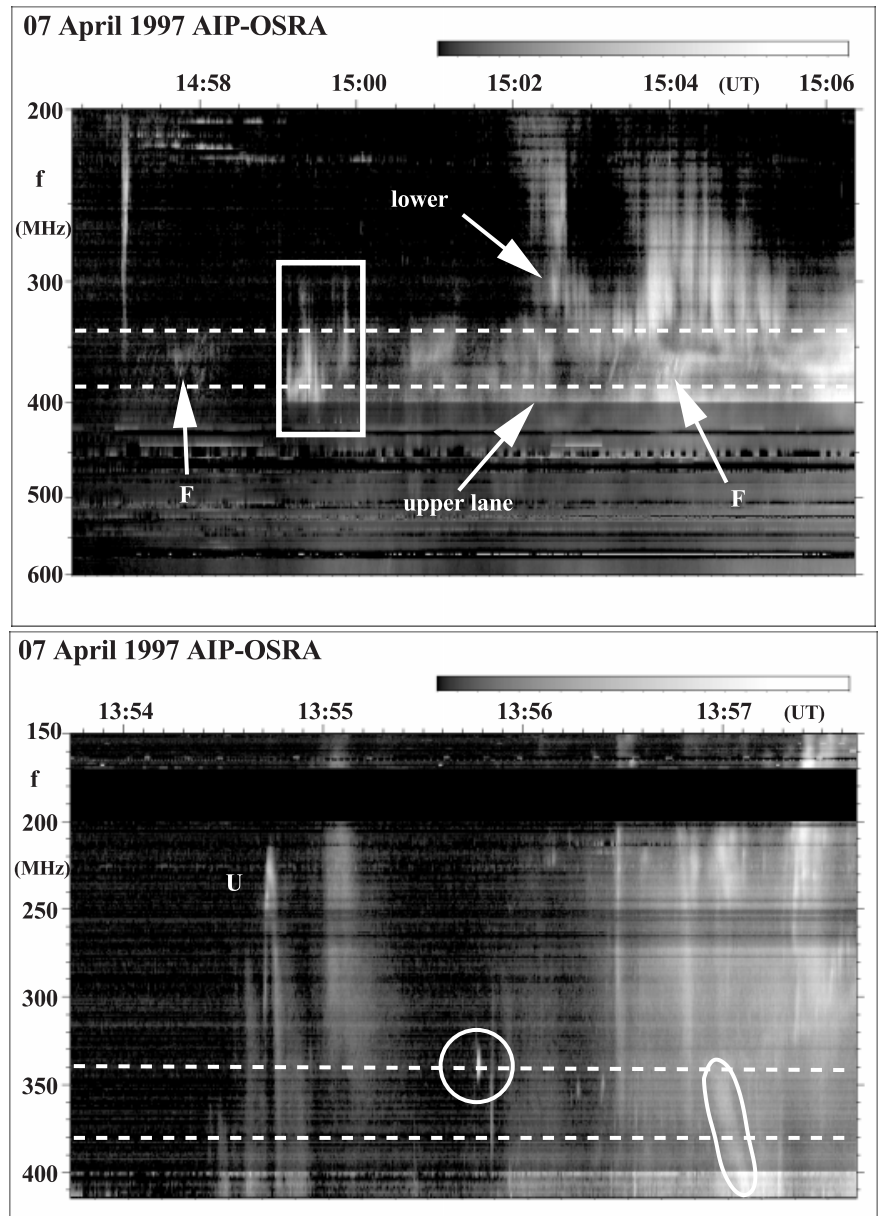

Fig. 4. In the top panel the onset stage of the termination shock (white box in Fig. 3) is shown. There is the arc pattern (box) which introduces the split band (Klassen et al. 1999). Stippled are the mean lower and upper split band frequencies of the termination shock. The same frequencies are also given in the bottom panel which is an enlargement of an early part of the main event (Fig. 2). The low frequency split band plays an extraordinary part here. It is the injection frequency of parts of the early nonthermal electron population. We have marked two characteristic burst patterns starting at this frequency. The type $\mathrm{U}$ bursts denoted in Fig. 2 are well visible.

the primary energy release site corresponds with about this frequency. This leads - having in mind the scheme Fig. 1 - to the assumption that we observe the lower outflow termination shock (the wave above the postflare loops) in the fundamental mode of plasma emission. No harmonic mode feature was found. There is also no low frequency feature which could be argued for being the upper outflow jet signature;

7. Between 420 and $700 \mathrm{MHz}$ the event is accompanied by at least three decimeter continuum patches with a characteristic duration of $5 \mathrm{~min}$ each. The patches have decaying bandwidth - the upper frequency boundary declines from 750 to $650 \mathrm{MHz}$ within $20 \mathrm{~min}$; 
8. Scattered over the termination shock signature but dominantly around 15:23 UT there are strong but short lived continuum brightenings (CB) ranging from 150 to about $400 \mathrm{MHz}$ (see Fig. 3 top, "CB"). These broad band "flashes" are mostly shorter than $1 \mathrm{~min}$ but rich in various continuum spectral fine structures.

The in many respects analogous characteristic of the analyzed emission to ordinary type II bursts indicates that it can be a radio signature of the lower reconnection outflow termination shock occuring in the late stage of the April 7, 1997 flare.

\section{On the Mach number in the outflow jet}

If the energy release in flares is regularly attributed to the fast reconnection process, then the supermagnetosonic outflowing jet should be a common feature. Why is the described radio signature not recognized more frequently? Why does it occur so late (almost one hour) after the impulsive phase in the considered event?

To answer these essential questions we have to ponder physical and observational arguments: does the deceleration region in the outflow jet permanently excite a type II-like radio signature? Do we always recognize and correctly interpret such a feature? The observational aspect we leave aside for the next section. Here we intend to discuss which conditions have to be fulfilled to obtain a type II radio signal from the outflow termination region. This means we have mainly to determine the Mach number in the reconnection outflow jet.

Bearing in mind the $\mathrm{H} \alpha$ and Yohkoh SXT observations, we assume that the April 7, 1997 event followed the dynamic flare scenario (see Fig. 1). When the current sheet formed below the erupting filament gets long enough the tearing instability sets in and the powerful energy release starts (Priest 1985, and references therein; see also Ugai 1987a; Forbes \& Malherbe 1991). Two pairs of SMSSs are formed bounding plasma jets outflowing from DR. The part of the current sheet below DR is shown enlarged in Fig. 5. The inflow region is denoted as "1", the downward ouflow jet between SMSSs as "2", and the region downstream of the perpendicular FMSS as "3". Notice that region " 2 " is downstream of the slow mode shocks but upstream of the fast mode shock.

In the stationary state the pressure of the hot plasma compressed between SMSSs is, roughly speaking, balanced by the plasma and magnetic field pressure in the inflow region. The outflow jet is accelerated to the velocity $v_{2}$ by the magnetic field tension of the strongly bended outflow field. The magnetosonic Mach number $M_{2}$ of the outflow plasma can be expressed as:

$M_{2}=\frac{v_{2}}{\sqrt{c_{\mathrm{S} 2}^{2}+v_{\mathrm{A} 2}^{2}}}$

where $c_{\mathrm{S} 2}=\sqrt{\gamma p_{2} / \rho_{2}}$ is the sound speed $\left(p_{2}\right.$ and $\rho_{2}$ are the pressure and the density of the outflowing plasma; $\gamma$ is the ratio of the specific heats hereinafter taken as

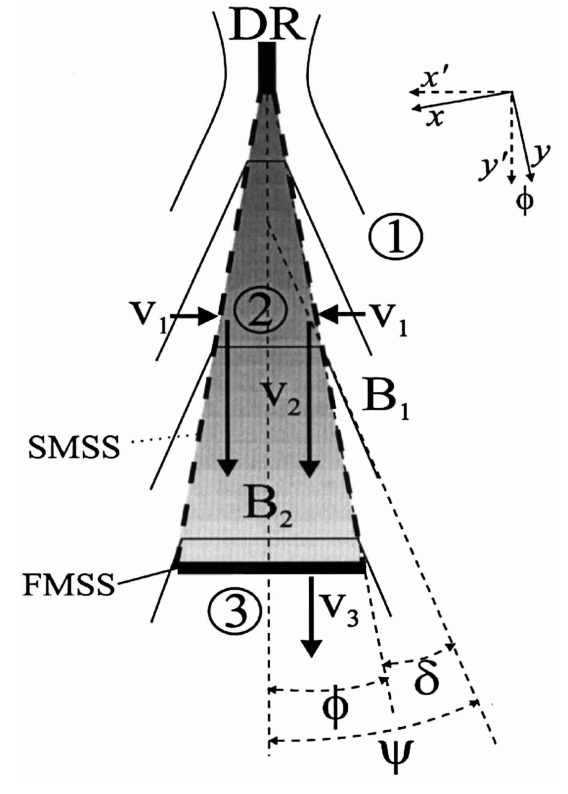

Fig. 5. The reconnection geometry, enlarged from Fig. 1. Slow mode standing shocks (SMSS) extending from the diffusion region (DR) are inclined by $\phi$ with respect to the axis of symmetry $\left(y^{\prime}\right)$. The plasma is inflowing from "1" at velocity $v_{1}$ into the shocks, carrying the field $B_{1}$ which is inclined to the $y^{\prime}$-axis by $\psi$. The tension of the bended field lines accelerates the plasma to speed $v_{2}$. A hot ouflow jet is formed between SMSSs (region "2") characterized by a weak perpendicular field $B_{2}$. If the jet becomes supermagnetosonic a fast mode standing shock (FMSS) is formed above the (slowly growing) postflare loops. Region "3" is downflow of FMSS. The coordinate system aligned with the SMSS is rotated by $\phi$ with respect to the $\left(x^{\prime}\right.$, $\left.y^{\prime}\right)$ system which is aligned with the ouflow jet $\left(v_{2}\right)$ and $B_{2}$.

$\gamma=5 / 3)$, and $v_{\mathrm{A} 2}=B_{2} / \sqrt{\rho_{2} \mu}$ is the Alfvén speed in the outflow region characterized by the weak field $B_{2}$. The velocity $v_{\mathrm{fms}}=\sqrt{c_{\mathrm{S} 2}^{2}+v_{\mathrm{A} 2}^{2}}$ is the fast magnetosonic speed perpendicular to the magnetic field $B_{2}$. Somewhere between DR and the postflare loop system below the reconnection region (Fig. 1) the perpendicular FMSS will be formed if the jet is characterized by $M_{2}>1$.

The relation between physical quantities in regions 1 and 2 are determined by "jump conditions" at the SMSS front (see, e.g., Priest 1982). The value of $M_{2}$ is in fact not governed by the conditions in the DR as usually considered (cf. Somov 1992, and references therein) but rather by the characteristics of the SMSS (see, e.g., Ugai 1999).

Bearing in mind the properties of the reconnection process and the symmetry of the problem there are some specific constraints in addition to the jump conditions (see Fig. 5):

1. The inflowing plasma velocity $v_{1}$ is relatively low - in the fast reconnection process it amounts to some $10 \%$ of the external Alfvén speed $v_{\mathrm{A} 1}$ (Ugai 1987a; see also Soward \& Priest 1982; Priest \& Forbes 1986).

2. The inclination $\phi$ of the SMSSs relative to the outflow direction $v_{2}\left(y^{\prime}\right.$-axis) is small; 
3. The outflowing magnetic field $B_{2}$ is inclined with respect to SMSS at the angle $\pi / 2-\phi$;

4. The angle $\psi$ between the inflowing magnetic field $B_{1}$ and the outflow $v_{2}\left(y^{\prime}\right.$-axis) is small, implying that the angle $\delta$ between $B_{1}$ and $\operatorname{SMSS}(\delta=\psi-\phi)$ is also small.

Applying the specific properties 2) and 3) to the jump conditions at the SMSS provides a specific family of solutions characterized by $v_{2} \perp B_{2}$. The number of free parameters is reduced - for a given plasma to magnetic pressure ratio upstream of $\operatorname{SMSS}\left(\beta\right.$ in region 1 , short $\beta_{1}$ ) the system is completely defined by specifying, e.g., the inflow speed $M_{\mathrm{A} 1}=v_{1} / v_{\mathrm{A} 1}$ and the inflowing magnetic field line inclination $\psi$.

An analysis of the complete set of equations is far beyond the scope of this paper. However, taking into account the items 1) and 4) the jump conditions at SMSSs can be written in an approximate form which provides an estimate of the outflow jet Mach number $M_{2}$. Following the procedure presented in the Appendix (see also Soward \& Priest 1982) the upper limit of the jet's Mach number can be estimated to $M_{2}=\sqrt{3}=1.73$ for the plasma to magnetic pressure ratio $\beta_{1} \ll 1$. This corresponds to a density jump at the FMSS of $X_{23}=\rho_{3} / \rho_{2}=2$ and a temperature ratio of $T_{3} / T_{2}=1.75$. These values are fairly consistent with those found in some of the numerical experiments (e.g. Forbes 1988). Notice that the heating effect of the strongest possible FMSS under the assumed conditions is less than a factor of 2 , which is not very impressive.

From previous considerations we notice that the FMSS, given our specific conditions $1-4$, can only be a relatively weak shock. Flare generated fast magnetosonic shocks are mostly weak shocks, too, but are well identified as sources of radio type II bursts (Fig. 5 in Mann et al. 1995, see also Nelson \& Melrose 1985). We expect that analogous radio emission is exited at the given FMSS. Assuming that the split of the emission band reflects the density jump between the upstream and downstream region (Vršnak et al. 2001 and references therein) one finds for the relative band split $B D W=\delta f / f=$ $\left(f_{\mathrm{d}}-f_{\mathrm{u}}\right) / f_{\mathrm{u}}=f_{\mathrm{d}} / f_{\mathrm{u}}-1$. Here $f_{\mathrm{d}}$ and $f_{\mathrm{u}}$ represent the frequencies of plasma emission from the FMSS upstream and downstream regions, respectively. Taking into account that $f \sim \sqrt{n}$, where $n$ is the electron density, one finds $B D W=\sqrt{n_{3} / n_{2}}-1=\sqrt{X_{23}}-1<0.41$ (see Appendix). On the other hand, using Eq. (16) and the maximum observed band split in the April 7, 1997 event $B D W \approx 0.2$, one finds the values $\beta_{1} \approx 0.3$ and $M_{2} \approx 1.3$. Here, we note a discrepancy: the Mach number is reasonable but the $\beta_{1}$ is seemingly too large. Furthermore, the value $\beta_{1}=0.3$ corresponds to a temperature jump of only $T_{2} / T_{1}=2.3$ (Eq. (12)) at SMSSs. This is much too low for flare conditions.

\section{Discussion and conclusion}

We have interpreted the "stationary type II burst" pattern as the radio signature of a standing fast mode termination shock in the reconnection outflow. Main arguments supporting the interpretation were: $(i)$ split band pattern (Fig. 3a); (ii) herringbones (Fig. 3b); (iii) arc pattern (Fig. 4); (iv) the source location relative to the flaring arcade observed by Yohkoh SXT, SOHO EIT, and the Nançay Radio Heliograph. We focus now on the problem of the rare identification of this phenomenon.

First, one must search for such a feature. Spontaneously, there is no reason to look for an energy release phenomenon of this kind so late after the impulsive phase. Further, such burst could well be considered as a next and independent flare event and thus not be studied in detail. On the other hand, there are already reports about special radio phenomena being isolated in spectrum and time in the main flare phase. Cliver (1983) reported on secondary peaks in solar microwave (and decimeter) outbursts. Aurass \& Kliem (1992) associated fine structures in "late decimeter and meter wave flare burst activation" with processes in the flaring current sheet leading to its final disruption. From our point of view both papers - basing on single frequency radio records - may have already described FMSS-associated emission features.

From observational experience we are not sure if the given phenomenon could be as easily identified if it happens in the impulsive flare phase. Here it would appear mixed with other flare burst radio sources. Additionally, necessary details of the spectrum are only accessible if the radio spectra are digitally recorded over a broad frequency band with a good time and spectral resolution. Such data were not generally available in earlier solar activity cycles. Furthermore our interest was pointed to this event also by the only recently available coronal imaging facilities SXT and EIT.

The phase of powerful energy release is accompanied by violent plasma motions. In such a turbulent environment it seems unlikely that the reconnection outflow could generate a stable and uniform FMSS all along the current sheet region in the extended arcade. Rather, it can be expected that the FMSS region is highly variable in time and space. Furthermore, the reconnection itself is probably taking place at different locations scattered all over the current sheet that is embedded in an entangled field line environment (see, e.g., Klimchuk 1996). Consequently, the band-split pattern probably would be smeared into continuum-like emission. Note that we have observed some minutes of broadband and fine structure-rich continuum emission during the proposed FMSS type II burst (the "CB" features in Fig. 3, top panel). If the emission would appear in the impulsive flare phase as non-split continuum enhancement no observer would associate it with a type II-like burst. Thus, radio signatures of FMSS could easily escape attention, or not be recognized at all, in early as well as in late phases of flares.

Finally, at least sometimes the impulsive phase of dynamical flares is caused by a distinct process, different from the main phase energy release sketched in Fig. 1. One example was presented by Aurass et al. (1999) where 
the impulsive phase was a result of a sequence of loop interactions in a highly sheared arcade. The loops reconnected into an unstable sigmoidal structure that erupted immediately after being formed. The two-ribbon phase of the flare was the aftermath. We note that the flare studied here was characterized by a sigmoidal pattern, too (Sterling et al. 2000).

Numerical simulations and analytical studies show that the reconnection outflow jet gets supermagnetosonic if certain conditions are fulfilled. The energy release by fast reconnection below the erupting filament is a complex process. It includes influences of heat conduction and chromospheric evaporation $^{3}$, as well as the effect of the strength of the magnetic field component perpendicular to the plane of reconnection ${ }^{4}$.

The favourable condition for obtaining a supermagnetosonic reconnection outflow is a low value of plasma to magnetic field pressure ratio $\beta_{1}$ in the inflow region (Eq. (14)). This value can be enhanced due to flareinduced chromospheric "evaporation" if reconnection does not occur too high in the corona. Such a "pollution" in the inflow region might result in the delayed (with respect to the impulsive phase) FMSS radio signature occurence.

A significant part can also be played by the ratio of the reconnecting component $B_{y}$ (defined in Fig. 5) and the transversal component of the magnetic field $B_{z}$ (Soward 1982). The Mach number of the reconnection outflow is smaller for a larger ratio $B_{z} / B_{y}$ (e.g. Forbes \& Malherbe 1986). The ratio $B_{z} / B_{y}$ is expected to decrease as the erupting filament stretches the initially sheared arcade structure, making the conditions for $M_{2}>1$ more favourable at later times, too.

In this context we notice that the downflow motions reported by McKenzie \& Hudson (1999) and McKenzie (2000) seem to be another type of motion since they are considerably slower than expected for an outflow jet. Those authors found velocities ranging between 100 and $500 \mathrm{kms}^{-1}$, whereas reconnection outflow speeds should be of the order of the coronal Alfvén velocity. The observed speeds are closer to velocities by which the magnetic islands formed by tearing are ejected along the current sheet (e.g. Forbes \& Priest 1983). Another explanation as proposed by McKenzie (2000) could be the already mentioned scenario attributed to Klimchuk (1996).

Finally, even if the FMSS is formed in the lower jet it does not necessarily lead to observable radio emission. The Mach number must be high enough so that the electric current associated with the FMSS is capable of exciting Langmuir turbulence (Kaplan \& Tsytovich 1973; Spicer \& Brown 1981, and references therein).

Now we can answer the questions posed in Sect. 3. Yes, the analyzed case is surely a special one because the effect is clearly visible in the spectral data. We presented some arguments possibly explaining why the radio signature of

\footnotetext{
${ }^{3}$ Forbes \& Malherbe (1986), Forbes et al. (1989); Yokoyama \& Shibata $(1998,2001)$.

4 Soward (1982), Forbes \& Malherbe (1986).
}

the outflow termination shock is a rare phenomenon and why it is probably associated with the late flare stage. Further we demonstrated how the effect can easily be overlooked in routine data inspection. On the other hand we found that a regime is possible where the standing type II signature and a fine structure rich continuum emission occur simultaneously. Finally we note that our estimates of the kinetic to magnetic pressure ratio of the instreaming plasma result in unreasonably large values (see also Forbes \& Malherbe 1991). This shows, beside the possibility that our interpretation is basically wrong, that there is still some fundamental discrepancy in the included aspects of a flare energy release reconnection model. We stress again that a possible solution is to invoke the $3-\mathrm{D}$ aspect of the problem and to take into account the complexity of coronal structures, proposed by Klimchuk (1996). We hope that our results help to stimulate further research in this field.

Acknowledgements. We thank the managers of the Yohkoh space mission and Nançay Multifrequency Radioheliograph Web pages, as well as to C. St. Cyr for the $1997 \mathrm{CME}$ table. Further we are grateful to W. Otruba and P. Schroll for the support in using Kanzelhöhe $\mathrm{H} \alpha$ data. The assistance of B. J. Thompson concerning SOHO EIT data and J. Khan concerning Yohkoh data was extremely useful. We thank H.-T. Claßen and H. Hudson for stimulating discussions. The comments of K. Shibata and another referee resulted in a substantial improvement of the paper.

\section{Appendix}

As pointed out in the Introduction the SMSSs are the basic large scale feature of the primary energy release process. Let us briefly inspect the relation between the characteristics of the outflow jet and the geometry of the SMSSs. We expect somewhat different results than obtained from the consideration of the DR itself which yields for the outflow velocity $v_{2} \approx B_{1}^{2} / \sqrt{\mu \rho_{2}}$ and for the compression $\rho_{2} / \rho_{1} \approx 5$ (Vršnak 1989, and references therein).

In the following, subscripts $x$ and $y$ denote the components of vectors in the coordinate system where the $y$-axis is aligned with the shock (Fig. 5). This means the $y$-axis is rotated with respect to the $y^{\prime}$ axis by the angle $\phi$. Since $\nabla \cdot B=0$ one finds:

$B_{x 1}=B_{x 2}$.

We assume that the flare evolution is comparatively slow so that the system can be described as a series of stationary states.

Then mass conservation implies:

$\rho_{1} v_{x 1}=\rho_{2} v_{x 2}$

where $\rho$ represents the mass density. Taking into account that $\phi$ and $\psi$ are small angles (see Sect. 3 and Fig. 5) one can take for the magnetic fields $B_{y 1} \gg B_{x 1}$ and $B_{x 2} \gg B_{y 2}$, and for the velocities $v_{x 1} \gg v_{y 1}$ and 
$v_{y 2} \gg v_{x 2}$. This implies $B_{1} \approx B_{1 y}, B_{2} \approx B_{2 x}, v_{1} \approx v_{1 x}$, and $v_{2} \approx v_{2 y}$.

The induction equation (see Priest 1982) reduces to:

$v_{y 2} B_{x 2} \approx v_{x 1} B_{y 1}$.

The jump conditions for the $y$ - and $x$-component of the equation of motion, and the energy equation (see Priest 1982) can be written in an approximate form:

$p_{2} \approx \frac{B_{y 1}^{2}}{2 \mu}+p_{1}$,

$\rho_{2} v_{x 2} v_{y 2} \approx \frac{B_{x 1} B_{y 1}}{\mu}$,

$\left(5 p_{2}+\rho_{2} v_{2}^{2}\right) v_{x 2} \approx\left(\frac{2 B_{y 1}^{2}}{\mu}+5 p_{1}\right) v_{x 1}$,

respectively. The ratio of specific heats is $\gamma=5 / 3$. Equation (6) can be written using Eqs. (2), (3), and (4) as:

$\rho_{1} v_{y 2}^{2} \approx \frac{B_{y 1}^{2}}{\mu}$.

Equation (8) implies $v_{2} \approx v_{2 y} \approx v_{\mathrm{A} 1}$ which can be substituted into Eq. (4) to get:

$B_{x 2} \approx B_{y 1} M_{\mathrm{A} 1}$,

where $M_{\mathrm{A} 1}=v_{1} / v_{\mathrm{A} 1}$. Equation (5) can be rewritten as:

$p_{2}=\frac{B_{y 1}^{2}}{2 \mu}\left(1+\beta_{1}\right)$,

where $\beta_{1}=2 \mu p_{1} / B_{1}^{2}$. Using Eqs. (3), (8), and (10) one finds from Eq. (7) the compression in the downstream region of SMSS (region "2" in Fig. 5):

$X_{12} \equiv \frac{\rho_{2}}{\rho_{1}}=\frac{5\left(1+\beta_{1}\right)}{2+5 \beta_{1}}$.

Dividing Eq. (10) with $p_{1}=n_{1} k T_{1}$ and using Eq. (11) one finds:

$T_{12} \equiv \frac{T_{2}}{T_{1}}=1+\frac{2}{5 \beta_{1}}$.

Using Eqs. (8), (9), and (10) the magnetosonic Mach number of the outflowing plasma (Eq. (1)) can be expressed as:

$M_{2}=\sqrt{\frac{X_{12}}{5\left(1+\beta_{1}\right) / 6+M_{\mathrm{A} 1}^{2}}}$.

Bearing in mind that the reconnection proceeds in the Petschek-like regime, i.e., $M_{\mathrm{A} 1}^{2} \ll 1$ can be applied, Eq. (12) can be written as:

$M_{2} \approx \sqrt{\frac{6 X_{12}}{5\left(1+\beta_{1}\right)}}=\sqrt{\frac{6}{2+5 \beta_{1}}}$,

where Eq. (11) was used. Equation (13) shows that there is an upper limit on the outflowing jet Mach number $M_{2}<$ $\sqrt{3}=1.73$ for $\beta_{1} \ll 1$. Furthermore, for a sufficiently high value of $\beta_{1}$ the flow becomes submagnetosonic and the termination shock will not be formed (see also Forbes \& Malherbe 1986, 1991). Substituting $M_{2}=1$ into Eq. (13) one finds the critical value $\beta_{1}^{\text {crit }}=4 / 5=0.8$.

Equation (13) provides an estimate of the compression in the FMSS formed above the postflare loop system (Figs. 1 and 5). Since in the jet $c_{\mathrm{s} 2} \gg v_{\mathrm{A} 2}$ is valid (implying $\beta_{2} \gg 1$ ) the compression $X_{23}$ at FMSS can be expressed by the hydrodynamical approximation (Priest 1982):

$X_{23} \approx \frac{4 M_{2}^{2}}{3+M_{2}^{2}}=\frac{8}{4+5 \beta_{1}}$.

Equation (14) shows that there is an upper limit on the compression at the FMSS $X_{23}=2$, which corresponds to the band splitting of $B D W=\sqrt{X_{23}}-1=0.41$. Finally, Eq. (14) can be expressed in terms of $B D W$ as:

$\beta_{1}=\frac{4}{5}\left(\frac{2}{(1+B D W)^{2}}-1\right)$,

where $B D W<\sqrt{2}-1$.

\section{References}

Aurass, H., Vršnak, B., Hofmann, A., \& Ruždjak, V. 1999, Sol. Phys., 190, 267

Aurass, H., \& Kliem, B. 1992, Sol. Phys., 141, 371

Cliver, E. W. 1983, Sol. Phys., 84, 347

Forbes, T. G. 1986, ApJ, 305, 553

Forbes, T. G. 1988, Sol. Phys., 117, 97

Forbes, T. G., \& Malherbe, J. M. 1986, ApJ, 302, L67

Forbes, T. G., \& Malherbe, J. M. 1991, Sol. Phys., 135, 361

Forbes, T. G., Malherbe, J. M., \& Priest, E. R. 1989, Sol. Phys., 120, 285

Forbes, T. G., \& Priest, E. R. 1982, Sol. Phys., 81, 303

Forbes, T. G., \& Priest, E. R. 1983, Sol. Phys., 84, 169

Kaiser, M. L., Reiner, M. J., Gopalswamy, N., et al. 1998, GRL, 25(14), 2501

Kaplan, S. A., \& Tsytovich, V. N. 1973, Plasma Astrophysics (Pergamon, Oxford)

Klassen, A., Aurass, H., Klein, K.-L., Hofmann, A., \& Mann, G. 1999, A\&A, 343, 287

Klassen, A., Aurass, H., Mann, G., \& Thompson, B. J. 2000, A\&AS, 141, 357

Klimchuk, J. 1996, Post-Eruption Arcades and 3-D Magnetic Reconnection, in Magnetic Reconnection in the Solar Atmosphere, ed. R. D. Bentley, \& J. T. Mariska, ASP Conf. Ser., 111, 319

Magara, T., Mineshige, S., Yokoyama, T., \& Shibata, K. 1996 ApJ, 466, 1054

Mann, G., Classen, H.-T., \& Aurass, H. 1995, A\&A, 295, 775

Masuda, S., Kosugi, T., Hara, H., Tsuneta, S., \& Ogawa, Y. 1994, Nature, 371, 495

McKenzie, D. E. 2000, Sol. Phys., 195, 381

McKenzie, D. E., \& Hudson, H. S. 1999, ApJ, 519, L93

Melrose, D. B. 1985, Plasma Emission Mechanisms, in Solar Radiophysics, ed. D. J. McLean, \& N. R. Labrum (Cambridge Univ. Press, Cambridge), 177

Nelson, G. S., \& Melrose, D. B. 1985, type II Bursts, in Solar Radiophysics, ed. D. J. McLean, \& N. R. Labrum (Cambridge Univ. Press, Cambridge), 333 
Nitta, S., Tanuma, S., Shibata, K., Maezawa, K. 2001, ApJ, 550,1119

Priest, E. R. 1982, Solar Magnetohydrodynamics (Reidel, Dordrecht)

Priest, E. R. 1985, Rep. Prog. Phys., 48, 955

Priest, E. R., \& Forbes, T. G. 1986, JGR, 91, 5579

Sato, T., \& Hayashi, T. 1979, Phys. Fluids, 22, 1189

Shibata, K., Masuda, S., Shimojo, M., et al. 1995, ApJ, 451, L83

Somov, B. V. 1986, A\&A, 163, 210

Somov, B. V. 1992, Physical Processes in Solar Flares, Astrophys. \& Space Sci. Lib. (Kluwer, Dordrecht), 172

Soward, A. M. 1982, J. Plasma Phys., 28, 415

Soward, A. M., \& Priest, E. R. 1982, J. Plasma Phys., 28, 335

Spicer, D. S., \& Brown, J. C. 1981, Solar Flare Theory, in The Sun as a Star, ed. S. Jordan (NASA SP-450), 413

Sterling, A. C., Hudson, H. S., Thompson, B. J., \& Zarro, D. M. 2000, ApJ, 532, 628
Thompson, B. J., Plunkett, S. P., Gurman, J. B., et al. 1998, GRL, 25, 2461

Tsuneta, S. 1996, ApJ, 456, 840

Ugai, M. 1987a, Phys. Fluids, 30, 2163

Ugai, M. 1987b, GRL, 14, 103

Ugai, M. 1992, Phys. Fluids B, 4, 2953

Ugai, M. 1999, JGR, 104, 6929

Vršnak, B. 1989, Sol. Phys., 120, 79

Vršnak, B., \& Lulić, S. 2000, Sol. Phys. 196, 157

Vršnak, B., Aurass, H., Magdalenić, J., \& Gopalswamy, N. 2001, A\&A, 377, 321

Yokoyama, T., Akita, K., Morimoto, T., Inoue, K., Newmark, J. 2001, ApJ, 546, L69

Yokoyama, T., \& Shibata, K. 1998, ApJ, 494, L113

Yokoyama, T., \& Shibata, K. 2001, ApJ, 549, 1160 